\title{
Limit on the Effective Quark Charge Radius from Inclusive ep Scattering at HERA
}

\author{
Oleksii Turkot ${ }^{* a}{ }$ Katarzyna Wichmann ${ }^{a}$ and Aleksander Filip Żarnecki ${ }^{b}$ \\ for the ZEUS Collaboration \\ ${ }^{a} D E S Y$, \\ ${ }^{b}$ Faculty of Physics, University of Warsaw \\ E-mail: oleksii.turkotedesy.de, katarzyna.wichmannedesy.de, \\ Filip.Zarneckiefuw.edu.pl
}

\begin{abstract}
The H1 and ZEUS experiments at HERA have recently presented the combined measurement of inclusive deep inelastic cross sections in neutral and charged current $e p$ scattering, based on the final data sample corresponding to a luminosity of about $1 \mathrm{fb}^{-1}$. The measurement was used as an input to QCD analyses, providing a set of parton distribution functions HERAPDF2.0. Here the analysis is extended to take into account possible signals from physics beyond the Standard Model. The accuracy of the data requires a combined analysis, taking into account possible contributions from the Beyond Standard Model processes in the QCD fit to the data. In the presented study this approach is developed for the quark form factor model and the resulting $95 \%$ C.L. limit for the radius of the electroweak charge of quarks is $0.45 \cdot 10^{-16} \mathrm{~cm}$.
\end{abstract}

The European Physical Society Conference on High Energy Physics

22-29 July 2015

Vienna, Austria

\footnotetext{
${ }^{*}$ Speaker.
} 


\section{Introduction}

Precision measurements of deep inelastic $e^{ \pm} p$ scattering (DIS) cross sections in the domain of large negative four-momentum-transfer squared, $Q^{2}$, allow searches for Beyond Standard Model (BSM) contributions far beyond the center-of-mass energy of the $e^{ \pm} p$ interactions. Due to virtual effects, the cross sections would be affected by new kinds of interactions where BSM particles would be exchanged. This could be leptoquarks, for which $e^{ \pm} p$ scattering is particularly sensitive, or gravitons in models with large extra dimensions. Far below the scale of the actual new physics, we can approximate all such BSM interactions as contact interactions, CI.

The analysis presented in this contribution focuses on the possible CI contribution due to finite quark radii, within the formalism of the quark form factor model [1]. Deviations of the observed cross section for $e p$ scattering at the highest available $Q^{2}$ from the SM predictions are searched for. In the ZEUS analysis [2] of the 1994-2000 HERA data, the uncertainties on the PDFs turned out to be a dominant source of systematics. However, as the CTEQ5D parameterization used for calculating model predictions, included only a small fraction of HERA data at low $Q^{2}$, one could assume it is not sensitive to BSM contributions. This is no longer the case in the analysis of the combined HERA measurements [3] as the parton distribution functions (PDFs) used to calculate model predictions are derived from the same data which is used to search for CI. For the analysis presented here a new procedure to set limits on the BSM model parameters was developed, where BSM contributions and QCD evolution are fitted simultaneously.

\section{Data samples}

The H1[4, 5, 6] and ZEUS [7] detectors were general purpose instruments which consisted of tracking systems surrounded by electromagnetic and hadronic calorimeters and muon detectors, ensuring close to $4 \pi$ coverage of the $e p$ interaction point. The presented study is based on the inclusive $\mathrm{NC}$ and $\mathrm{CC}$ cross-section measurements for unpolarised $e^{ \pm} p$ scattering at HERA, resulting from the combination [3] of the all available data from the H1 and ZEUS experiments. The analysis included 41 different data sets collected at four different centre-of-mass energies from 1994 to 2007 (HERA I and HERA II). During the HERA I period, each experiment collected about $100 \mathrm{pb}^{-1}$ of $e^{+} p$ and $15 \mathrm{pb}^{-1}$ of $e^{-} p$ data, while at HERA II period, about $150 \mathrm{pb}^{-1}$ of $e^{+} p$ and $235 \mathrm{pb}^{-1}$ of $e^{-} p$ data were added. The original double-differential cross-section measurements, as published by the H1 and ZEUS collaborations, were averaged using the HERAverager tool [8] which is based on a $\chi^{2}$ minimisation method. The 2927 published cross-section values were combined to 1307 averaged cross-section measurements. For the resulting 1620 degrees of freedom, a $\chi_{\min }^{2}=1687$ was obtained, demonstrating very good consistency of all considered data sets. Since H1 and ZEUS used different reconstruction methods, similar systematic sources influenced the measured cross sections differently. Therefore, requiring the cross sections to agree at all $x$ and $Q^{2}$ values resulted in significant reduction of the systematic uncertainties. For more details, see [3].

\section{QCD analysis}

In the following, the framework used in the perturbative QCD (pQCD) analysis of the combined data, as used in the HERAPDF2.0 study [3], is briefly described. Only cross sections for $Q^{2}$ 
starting from $Q_{\min }^{2}=3.5 \mathrm{GeV}^{2}$ were used in the analysis. A fit to the data, resulting in the set of PDFs, was obtained by solving the DGLAP evolution equations at NLO in the $\overline{\mathrm{MS}}$ scheme. This was done using the programme QCDNUM [9] within the HERAFitter framework [10]. The PDFs parameterised are the gluon distribution, $x g$, the valence-quark distributions, $x u_{v}, x d_{v}$, and the $u$ type and $d$-type anti-quark distributions, $x \bar{U}, x \bar{D}$. The PDFs parameter values at the starting scale $\mu_{\mathrm{f}_{0}}^{2}$ were fitted to the data, taking into account statistical uncertainties, as well as uncorrelated and correlated systematic uncertainties of the input experimental data.

Uncertainties of the PDFs resulting from the experimental uncertainties were determined with the criterion $\Delta \chi^{2}=1$ and verified with the Monte Carlo method based on analysis of a large number of pseudo data sets called replicas. The two approaches gave consistent estimates of experimental errors. The uncertainties on HERAPDF2.0 due to the choice of the model settings and the form of the parameterisation were also evaluated, and the total PDF uncertainty was obtained by adding in quadrature the experimental, the model and the parameterisation uncertainties. For more details, see [3].

\section{Quark form factor}

One of the possible methods to search for deviations from SM predictions in $e p$ scattering is to assign a finite size for the radius of the electroweak charges of electrons and/or quarks while assuming the SM gauge bosons remain pointlike and their couplings are unchanged. The expected suppression of the SM cross section can be described using a semi-classical form factor approach. If the expected deviations are small, the SM predictions for the cross sections are modified, approximately, to:

$$
\frac{d \sigma}{d Q^{2}}=\frac{d \sigma^{S M}}{d Q^{2}}\left(1-\frac{R_{e}^{2}}{6} Q^{2}\right)^{2}\left(1-\frac{R_{q}^{2}}{6} Q^{2}\right)^{2}
$$

where $R_{e}$ and $R_{q}$ are the root-mean-square radii of the electroweak charge of the electron and the quark, respectively. In the present analysis only the possible finite spatial distribution of the quark charge is considered and the electron is assumed to be point-like $\left(R_{e} \equiv 0\right)$.

The QCD analysis described in the previous section is extended by introducing $R_{q}$ as additional model parameter and modifying all $e^{ \pm} p$ DIS cross-section predictions according to formula (4.1), to take into account possible signals from physics beyond SM. The estimate of the quark radius squared resulting from the simultaneous fit of $R_{q}$ and the PDF parameters to the data is

$$
R_{q}^{2 \text { Data }}=-0.5 \cdot 10^{-6} \mathrm{GeV}^{-2}
$$

The result is in good agreement with the SM predictions, taking into account the expected statistical fluctuations. As the obtained value is negative, outside the considered $R_{q}^{2}$ range, we use the value of $R_{q}^{2 \text { Data }}=0$ for the limit setting. This corresponds to the assumption that, within the considered model, the cross section enhancement at high $Q^{2}$ observed in the data could only be due to statistical fluctuations. 


\section{Limit setting procedure}

The limit on the effective quark radius $R_{q}$ is derived by using a frequentist approach. We compare the most likely value of the quark radius squared, determined from the $\chi^{2}$ minimization, for the actual input data and for a large number of equivalent replicas. Excluded at the 95\% C.L. are $R_{q}$ values which, in more than $95 \%$ of the replicas, result in the fitted radius squared value, $R_{q}^{2 \text { Fit }}$, greater than that obtained for the data, $R_{q}^{2 \text { Data }}$.

To set the limit, a distribution of $R_{q}^{2 \text { Fit }}$ values had to be reconstructed from QCD fits to multiple replicas, for different values of the assumed true radius, $R_{q}^{\text {true }}$. However, the assumed value of $R_{q}^{\text {true }}$ needs to be taken into account in the replicas generation as well. Therefor, for each $R_{q}^{\text {true }}$ value considered, a dedicated QCD fit to the combined data was first performed (with $R_{q}$ fixed to $R_{q}^{\text {true }}$ ) to obtain the corresponding PDF parametrization. These PDFs were then used to generate multiple data replicas for a given value $R_{q}^{\text {true }}$. Replica data-sets were created by taking the reduced cross sections calculated from PDFs and fluctuating their values randomly within given statistical and systematic uncertainties taking into account correlations. All uncertainties were assumed to follow the Gaussian distribution. Finally, the full QCD fit was performed on the generated replica set using a $\chi^{2}$ method, to reconstruct the $R_{q}^{2 \text { Fit }}$ distribution.

In the last step, the probability of obtaining the $R_{q}^{2}$ Fit value smaller than that obtained for the actual data, $\operatorname{Prob}\left(R_{q}^{2 \text { Fit }}<R_{q}^{2 \text { Data }}\right)$, was plotted as a function of $R_{q}^{\text {true }}$, as shown in Fig. 1. Points with error bars represent replica sets generated for subsequent $R_{q}^{\text {true }}$ values. The errors are statistical only, calculated assuming a binomial probability distribution for $\operatorname{Prob}\left(R_{q}^{2 \text { Fit }}<R_{q}^{2 \text { Data }}\right)$. The blue dashed line, drawn to guide the eye, represents the dependence expected for gaussian distribution of $R_{q}^{2 \text { Fit }}$. For comparison, the dependence obtained with PDF parameters fixed to HERAPDF2.0 values (both in replica generation and in $R_{q}$ fits to replica sets based on $\chi^{2}$ minimization), is indicated by the green dash-dotted line. The probability distribution is interpolated to calculate the $R_{q}$ value corresponding to the $95 \%$ C.L. limit. The interpolation is done with a fit of an exponential function to the points with an estimated probability between 2.5 and $8.5 \%$.

\section{Results}

The limit setting procedure described above was repeated for different model and parameter settings considered as systematic checks in the QCD fitting procedure (see [3] for details). The resulting variations of the $R_{q}$ limit values are very small, of the order of $1 \%$. The obtained $95 \%$ C.L. upper limit on the effective quark-charge radius is:

$$
R_{q}<0.45 \cdot 10^{-16} \mathrm{~cm} .
$$

This result is about $10 \%$ weaker than the limit obtained without taking into account possible $R_{q}$ influence on the PDF fit result. As a cross check the limit on $R_{q}$ was also determined from the simultaneous PDF and $R_{q}$ fit to the data (see Sec. 4 and 5), looking at the variation of the $\chi^{2}$ value minimized with respect to the PDF parameters when changing the $R_{q}$ value. The estimated limit was in a good agreement with the result presented here.

Cross section deviations corresponding to the $95 \%$ C.L. exclusion limit for the effective radius, $R_{q}$, of the electroweak charge of the quark are compared with the combined H1 and ZEUS high- $Q^{2}$ NC DIS data in Fig. 2. 


\section{Conclusions}

The H1 and ZEUS combined measurement of inclusive deep inelastic cross sections in neutral and charged current $e^{ \pm} p$ scattering, was used to set limit on the possible physics beyond SM in the classical quark form factor approximation. The QCD analyses of HERA data, providing a set of parton distribution functions HERAPDF2.0, was extended to take into account possible cross section modification due to a finite quark charge radius. As the same data are used to calculate PDFs and to set limit on BSM scenario, the limit setting procedure was based on a simultaneous fit of PDF parameters and $R_{q}$, properly taking into account possible contributions from the BSM processes in the QCD fit to the data.

A quark-charge radius larger than $0.45 \cdot 10^{-16} \mathrm{~cm}$ has been excluded on a $95 \%$ C.L., assuming $R_{e}=0$, which is almost a factor of two improvement compared to the published ZEUS limit of $0.85 \cdot 10^{-16} \mathrm{~cm}$, based on the HERA I data [2]. The present result improves the limit set in $e p$ scattering by the $\mathrm{H} 1$ collaboration [11] $\left(R_{q}<0.65 \cdot 10^{-16} \mathrm{~cm}\right)$ and is similar to the limit presented by the $\mathrm{L} 3$ collaboration $\left(R_{q}<0.42 \cdot 10^{-16} \mathrm{~cm}\right.$, assuming $\left.R_{e}=0\right)$, based on a quark-pair production measurement at LEP2 [12].

\section{References}

[1] G. Kopp, D. Schaile, M. Spira and P.M.Zerwas, Bounds on radii and magnetic dipole moments of quarks and leptons from LEP, SLC and HERA, Z. Phys. C65 (1995) 545.

[2] ZEUS Coll., S. Chekanov et al., Search for contact interactions, large extra dimensions and finite quark radius in ep collisions at HERA, Phys. Lett. B 591 (2004) 23.

[3] H1 ans ZEUS Collaborations, Combination of Measurements of Inclusive Deep Inelastic $e^{ \pm} p$ Scattering Cross Sections and QCD Analysis of HERA data, DESY-15-039, arXiv: 1506.06042.

[4] I. Abt et al., The H1 detector at HERA, Nucl. Instr. Meth. A 386 (1997) 310.

[5] I. Abt et al., The Tracking, calorimeter and muon detectors of the H1 experiment at HERA, Nucl. Instr. Meth. A 386 (1997) 348.

[6] R.D. Appuhn et al., Hl backward upgrade with a SPACAL calorimeter: The Hadronic section, DESY-96-013 (1996).

[7] ZEUS Collaboration, U. Holm (ed.), The ZEUS Detector. Status Report, DESY 1993.

[8] The documentation and the package can be found at: https://wiki-zeuthen. desy.de/HERAverager

[9] M. Botje, QCDNUM: Fast QCD evolution and convolution, Comp. Phys. Comm. 182 (2011) 490.

[10] S. Alekhin et al., HERAFitter, Open Source QCD Fit Project, arXiv:1410.4412.

[11] H1 Coll., F.D. Aaron et al., Search for Contact Interactions in $e^{ \pm} p$ Collisions at HERA, Phys. Lett. B 705 (2011) 52.

[12] L3 Coll., M. Acciarri et al., Search for manifestations of new physics in fermion pair production at LEP, Phys. Lett. B 489 (2000) 81. 


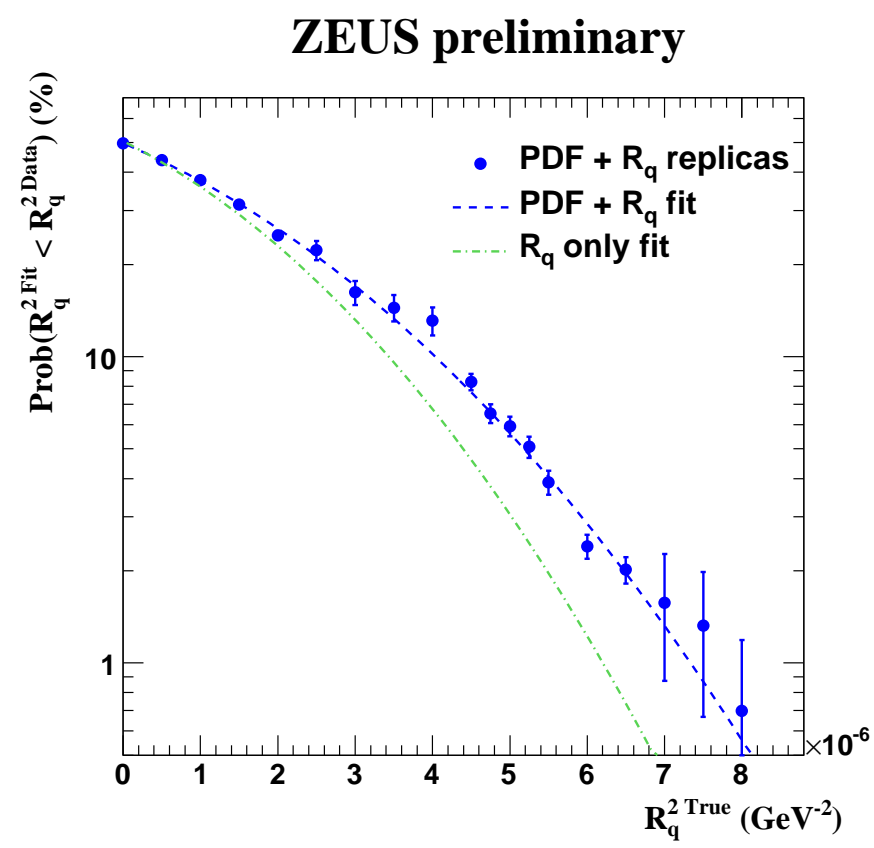

Figure 1: The probability of obtaining the $R_{q}^{2 \text { Fit }}$ value smaller than that obtained for the actual data, $R_{q}^{2 \text { Data }}$, calculated from multiple Monte Carlo replicas, as a function of the assumed value for the quark radius, $R_{q}^{\text {true }}$. Points represent Monte Carlo replica sets generated for different values of $R_{q}^{\text {true }}$, with $R_{q}^{2 \text { Fit }}$ values estimated from the simultaneous fit of the $R_{q}$ and PDF parameters. The green dash-dotted line represents the dependence obtained when fixing the PDF parameters to HERAPDF2.0 values.
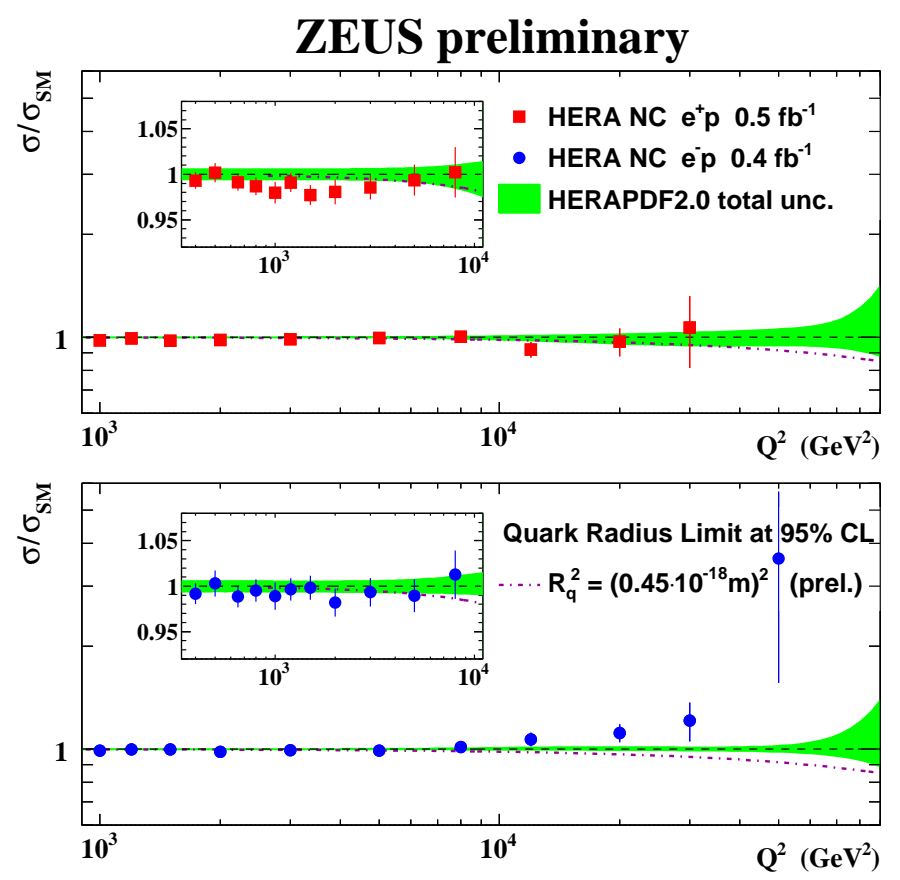

Figure 2: Combined $\mathrm{H} 1$ and ZEUS $e^{+} p$ data (top plot) and $e^{-} p$ data (bottom plot) compared with the 95\% C.L. exclusion limit for the effective mean-square radius of the electroweak charge of the quark. Results are compared to the Standard Model expectations calculated using the HERAPDF2.0 parton distributions. The green bands represent the total uncertainty on the HERAPDF2.0 predictions. The insets show the comparison in the $Q^{2}<10^{4} \mathrm{GeV}^{2}$ region, with a linear ordinate scale. 